\title{
Nonlinear Magnetostatic Finite-Element Formulation for Models with Radial Symmetry
}

\author{
Dries Vanoost ${ }^{1}$, Herbert De Gersem ${ }^{2,3}$, Joan Peuteman ${ }^{1,4}$, Georges Gielen ${ }^{5}$, Davy Pissoort ${ }^{1,5}$ \\ ${ }^{1}$ KU Leuven Kulab, ReMI Research Group, Oostende, Belgium \\ ${ }^{2}$ KU Leuven Kulak, Wave Propagation and Signal Processing Research Group, Kortrijk, Belgium \\ ${ }^{3}$ KU Leuven, Department of Physics and Astronomy, Solid-State Physics and Magnetism, Heverlee, Belgium \\ ${ }^{4}$ KU Leuven, Department of Electrical Engineering, Electrical Energy and Computer Architecture, Heverlee, Belgium \\ ${ }^{5} \mathrm{KU}$ Leuven, Department of Electrical Engineering, Microelectronics and Sensors, Heverlee, Belgium
}

\begin{abstract}
This paper discusses the implementation of nonlinear material properties in a two-dimensional (2D) magnetostatic finite-element (FE) solver for radially symmetric models. It is shown that, when saturation occurs, the particular dependence of the magnetic vector potential on the radial coordinate as required for radial symmetry, is lost. Despite the 2D geometrical symmetry, the weighted residual approach requires numerical quadrature points to be distributed along the direction of symmetry. The 2D solver with radial symmetry is illustrated by calculating the performance of a dual-rotor permanent-magnet axial-flux machine.
\end{abstract}

Index Terms-Finite element methods, magnetostatics, nonlinear magnetics, partial differential equations, permanent magnet machines.

\section{INTRODUCTION}

$\mathbf{E}$ FFICIENT use of ferromagnetic cores in electromagnetic devices requires the material to be used at early saturation [1]. Such systems are described by the magnetostatic or magnetoquasistatic subsets of the Maxwell equations. In the presence of saturable materials, the formulations are linearized by a fixed-point technique, e.g. the polarization method [2], the successive substitution technique [3] or the Newton method [4]. Nonlinear problems come with a significant increase of the computational cost. 2D simulation is favoured over 3D simulation whenever the geometry, excitations and boundary conditions are in essence two-dimensional or when a reduction to a $2 \mathrm{D}$ model introduces a tolerable modelling error. Cartesian and axisymmetric 2D models are well known and are provided in both commercial and freeware software packages. A radially symmetric situation is characterized by magnetic flux lines confined in cylindrical shells and by currents in radial direction. A 2D reduction for this case is less obvious, but is promising for an important segment of electromagnetic devices, e.g., axial-flux machines [5], disk motors, cylindrical magnetic brakes and multi-coil induction cooking systems. Previous research [6], [7] has shown that the choice of dedicated finite-element (FE) shape functions is of paramount importance to guarantee the partition-ofunity, consistency and convergence properties of the numerical scheme. In particular, the FE shape functions need to depend on the radial coordinate. Such dependence on the direction of symmetry is not encountered in the cartesian and axisymmetric cases. In this paper, the 2D FE solver for radial symmetry is equipped with the Newton method for dealing with nonlinear materials. The particularity of radial symmetry necessitates a specific nonlinear update scheme and construction of the Jacobian matrices.

Manuscript received XXX, XXXX. Corresponding author: Dries Vanoost (e-mail: Dries.Vanoost@kuleuven-kulak.be)

Digital Object Identifier inserted by IEEE

\section{Nonlinear Formulation And Newton Procedure}

The magnetostatic formulation

$$
\nabla \times(\nu(\mathbf{B}) \nabla \times \mathbf{A})=\mathbf{J}
$$

is obtained from Ampère's equation by relating the magnetic flux density $\mathbf{B}=\nabla \times \mathbf{A}$ to the magnetic vector potential $\mathbf{A}$ and relating the magnetic field strength $\mathbf{H}$ to the magnetic flux density by $\mathbf{H}=\nu \mathbf{B}$. Here, the reluctivity $\nu$ depends on the magnetic flux density because of ferromagnetic saturation. Equation (1) is linearized by the Newton method [4]. The most common form solves for increments $\delta \mathbf{A}$ of $\mathbf{A}$. It is, however, beneficial to explicitly linearize the problem for each material point in its operating point $\left(\mathbf{H}^{(k)}, \mathbf{B}^{(k)}\right)$ [8], i.e.,

$$
\mathbf{H}(\mathbf{B})=\mathbf{H}_{\mathrm{m}}^{(k)}+\overline{\bar{\nu}}_{\mathrm{d}}^{(k)} \mathbf{B},
$$

where $\mathbf{H}_{\mathrm{m}}^{(k)}$ is the magnetisation field strength and $\overline{\bar{\nu}}_{\mathrm{d}}^{(k)}$ is the differential reluctivity tensor. Equation (2) serves as linearization of any isotropic or anisotropic, hard or soft magnetic material. E.g., for an isotropic steel with a scalar $\mathrm{BH}$-characteristic (Fig. 1), the linearized characteristic is described by

$$
\begin{aligned}
\mathbf{H}_{\mathrm{m}}^{(k)} & =H_{\mathrm{m}}^{(k)} \mathbf{e}_{B}^{(k)} ; \\
\overline{\bar{\nu}}_{\mathrm{d}}^{(k)} & =\nu^{(k)} \overline{\overline{1}}+2 \mathbf{B}^{(k)}\left(\frac{\mathrm{d} \nu}{\mathrm{d} B^{2}}\right)^{(k)} \mathbf{B}^{(k)},
\end{aligned}
$$

where $\overline{\overline{1}}$ is the unit tensor, $\mathbf{e}_{B}^{(k)}=\mathbf{B}^{(k)} /\left|\mathbf{B}^{(k)}\right|$ and $H_{\mathrm{m}}^{(k)}, \nu^{(k)}$ and $\left(\frac{\mathrm{d} \nu}{\mathrm{d} B^{2}}\right)^{(k)}$ are evaluated for the $B H$-characteristic in point $\left.\left(\left|\mathbf{H}^{(k)}\right|,\left|\mathbf{B}^{(k)}\right|\right)\right)$. The Newton procedure amounts to solving $\mathbf{A}^{(k+1)}$ from

$$
\nabla \times\left(\overline{\bar{\nu}}_{\mathrm{d}}^{(k)} \nabla \times \mathbf{A}^{(k+1)}\right)=\mathbf{J}-\nabla \times \mathbf{H}_{\mathrm{m}}^{(k)} .
$$

The iterations are stopped when a convergence criterion is met, typically when the relative change in the stored magnetic energy is below a user-defined threshold. 


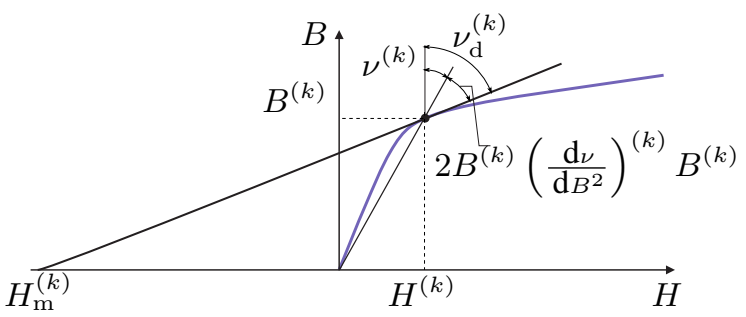

Fig. 1: $B H$-characteristic with operating point $\left(H^{(k)}, B^{(k)}\right)$, chord reluctivity $\nu^{(k)}$, magnetisation field strength $H_{\mathrm{m}}^{(k)}$, differential reluctivity $\nu_{\mathrm{d}}^{(k)}$ and difference reluctivity $2 B^{(k)}\left(\frac{\mathrm{d} \nu}{\mathrm{d} B^{2}}\right)^{(k)} B^{(k)}$.

\section{REDUCTION TO RADIAL SYMMETRY}

A cylindrical coordinate system $(r, \theta, z)$ with $r$ the radial, $\theta$ the peripheral and $z$ the axial coordinate is used. A model is said to feature radial material symmetry when the material distribution does not depend on $r$. When the current density $\mathbf{J}=\left(J_{r}, 0,0\right)$ has only a radial component, the generated magnetic flux density $\mathbf{B}=\left(0, B_{\theta}, B_{z}\right)$ will only have a peripheral and an axial component [9], [6], [10]. Moreover, $J_{r}$ and $B_{\theta}$ are inversely proportional to $r$, whereas $B_{z}$ does not depend on $r$. The current density is expressed more specifically by $J_{r}=H_{\mathrm{j}}(\theta, z) / r$. The specific dependence of the fields on $r$ is called radial field symmetry [11]. In the standard cartesian and axisymmetric cases, the material and field symmetries are of the same type, whereas in the radially symmetric case, as well as in the helicoidally symmetric case [11], this is no longer true.

The axial-flux machine of Fig. 2 serves as an illustration. The magnetically active part of the machine has a radially symmetric geometry for $r_{1} \leq r \leq r_{2}$ and carries a radially oriented current density. The symmetry is violated by the coil end parts and by fringing effects. However, these end effects are assumed to have a negligible influence on the fields in $r_{1} \leq r \leq r_{2}$. The magnetic flux lines are confined on cylindrical shells. It makes sense to consider a 2D model ranging between $r=r_{1}$ and $r=r_{2}$, with a radial extent $\ell_{r}=r_{2}-r_{1}$ and located at a reference shell $S_{\text {eq }}$ with reference radius $r_{\text {eq }}$. The magnetic vector potential can be reduced to its radial component $A_{r}$.

\section{Finite-Element Discretisation}

It is not recommended to explicitly restrict the vectorial partial differential equation (1) to its $r$-component and then introduce nodal FE shape functions for $A_{r}$. A better technique is to define a transformation between the radially symmetric model and a cartesian or axisymmetric model [12], [10]. As another alternative, we suggest to define dedicated vectorial FE shape functions. The lowest-order FE shape functions $\mathbf{w}_{e, j}$ defined at element $e$ read:

$$
\begin{aligned}
\mathbf{w}_{e, j} & =\frac{N_{e, j}(r, \theta, z)}{r_{2}-r_{1}} \mathbf{e}_{r} ; \\
N_{e, j} & =\frac{a_{e, j} \frac{1}{r}+b_{e, j} r \theta+c_{e, j} \frac{z}{r}}{2 S_{e}},
\end{aligned}
$$

where $\mathbf{e}_{r}$ is the radial unit vector, $S_{e}$ is the cross-sectional area of the considered triangle in the reference plane, and

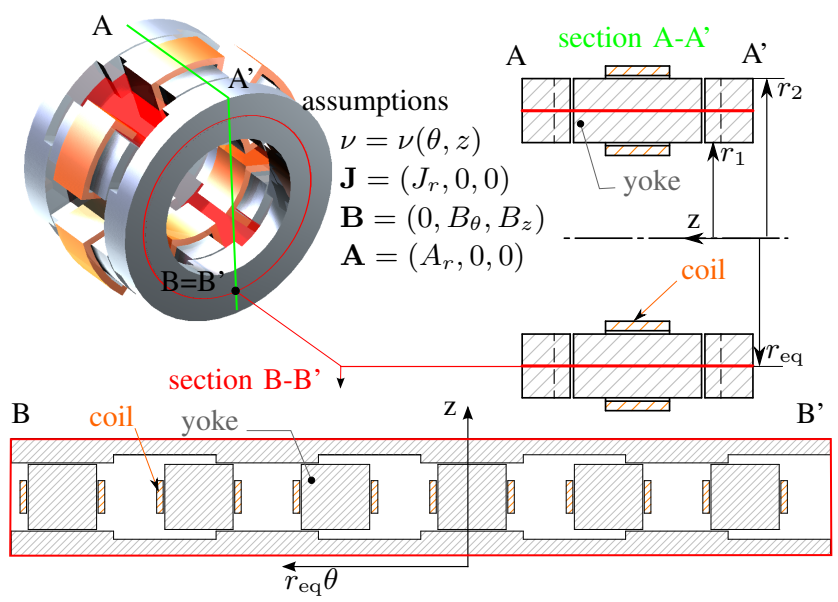

Fig. 2: 3D model of an axial-flux machine; diametric section A-A'; cylindrical section B-B' stratified to a $2 \mathrm{D}$ plane.

the coefficients $a_{e, j}, b_{e, j}$ and $c_{e, j}$ follow from enforcing $N_{e, j}\left(r_{\mathrm{eq}}, \theta_{q}, z_{q}\right)=\delta_{j q}$ with $\delta$ the Kronecker delta function at the nodes in the reference plane. In [6], it has been shown that $\mathbf{w}_{e, j}(r, \theta, z)$ are edge elements [13], fulfil a partition-of-unity property, are consistent and guarantee polynomial convergence of the discrete solution.

The FE shape functions (6) are used to weigh the linearised formulation and to discretise the magnetic vector potential, leading to the discrete counterpart of (5), i.e.,

$$
\mathbf{K}_{\nu_{\mathrm{d}}}^{(k)} \widehat{\mathbf{a}}^{(k+1)}=\widehat{\mathbf{j}}+\widehat{\mathbf{j}}_{\mathrm{m}}^{(k)},
$$

where the notation is borrowed from the finite integration technique [14] and the matrix and vector entries are:

$$
\begin{aligned}
\mathbf{K}_{\nu_{\mathrm{d}}, i j}^{(k)} & =\int_{V}\left(\overline{\bar{\nu}}_{\mathrm{d}}^{(k)} \nabla \times \mathbf{w}_{i}\right) \cdot\left(\nabla \times \mathbf{w}_{j}\right) \mathrm{d} V ; \\
\widehat{\mathbf{j}}_{i} & =\int_{V} \mathbf{J} \cdot \mathbf{w}_{i} \mathrm{~d} V ; \\
\widehat{\mathbf{j}}_{\mathrm{m}, i}^{(k)} & =-\int_{V} \mathbf{H}_{\mathrm{m}}^{(k)} \cdot\left(\nabla \times \mathbf{w}_{i}\right) \mathrm{d} V .
\end{aligned}
$$

\section{UPDATE FOR THE NONLINEARITY}

The magnetic flux density depends on the radial coordinate $r$. As a consequence, also the saturation level depends on $r$. Material points $\left(r_{a}, \theta_{p}, z_{p}\right)$ at a shell with a small radius $r_{a}$ saturate earlier than corresponding material points $\left(r_{b}, \theta_{p}, z_{p}\right)$ at a larger radius $r_{b}$. Evaluating the material characteristic at a single material point, e.g., at the reference shell, may lead to a poor treatment of the nonlinearity. Instead, a number of material points $\left(r_{p}, \theta_{p}, z_{p}\right)$ are distributed along the radial direction according to the points used by the numerical quadrature rule for calculating (9), (10) and (11). At each material point, the magnetic flux density is calculated on the basis of the results of the previous Newton step, the material characteristic is evaluated and the magnetisation field strengths $\mathbf{H}_{\mathrm{m}, p}^{(k)}$ and the differential reluctivity tensors $\overline{\bar{\nu}}_{\mathrm{d}, p}^{(k)}$ are determined. The fact that $\overline{\bar{\nu}}_{\mathrm{d}}^{(k)}$ and $\mathbf{H}_{\mathrm{m}}^{(k)}$ depend on $r$ substantially complicates the calculation of (9) and (11) compared to the linear case reported in [6]. Such situation is unseen in standard cartesian and axisymmetric 2D formulations. 


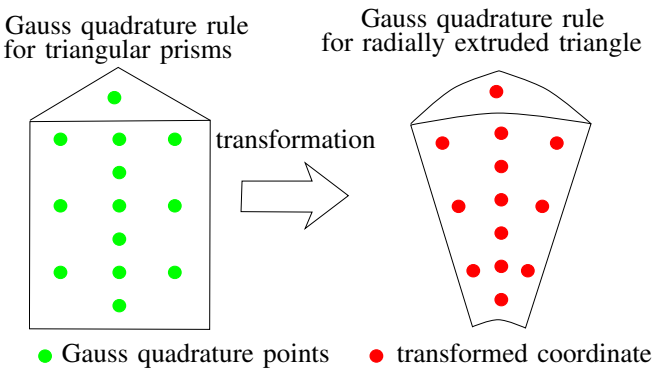

Fig. 3: Gauss quadrature points in a triangular prism and transformation of the domain to a tapered triangular prism.

The integration domain $\cup_{e} V_{e}=V$ is decomposed in tapered triangular prisms $V_{e}=S_{e} \otimes\left[r_{1}, r_{2}\right]$. The integrals in (9), (10) and (11) are calculated per element $V_{e}$. A first approach consists of mapping the integrands from $V_{e}$ to a reference prism and using 3D Gauss quadrature rules specified for such prisms [15] (Fig. 3). However, in [7], it was shown that the inverse proportionality on $r$ hampers the convergence of Gauss-type quadrature rules. Instead of invoking more accurate but also more expensive adaptive integration techniques, here, the integrals are worked out in two successive steps, i.e., the integration in $r$ is carried out numerically, whereas the integration in $\theta$ and $z$ is done analytically. This results in

$$
\begin{aligned}
\mathbf{K}_{\nu_{\mathrm{d}}, e, i j}^{(k)} & =\frac{1}{4 S_{e} \ell_{r}}\left[\begin{array}{ll}
\frac{c_{e, i}}{r_{\mathrm{eq}}} & -b_{e, i}
\end{array}\right] \overline{\bar{\nu}}_{\mathrm{d}, \mathrm{av}, e}^{(k)}\left[\begin{array}{c}
\frac{c_{e, j}}{r_{\mathrm{eq}}} \\
-b_{e, j}
\end{array}\right] \\
\widehat{\mathbf{j}}_{e, i} & =\frac{S_{e}}{3} \frac{H_{\mathrm{j}}}{r_{\mathrm{eq}}} ; \\
\widehat{\mathbf{j}}_{\mathrm{m}, e, i} & =-\frac{1}{2}\left[\begin{array}{ll}
\frac{c_{e, i}}{r_{\mathrm{eq}}} & -b_{e, i}
\end{array}\right]\left[\begin{array}{c}
H_{\mathrm{m}, \mathrm{av}, e, \theta}^{(k)} \\
H_{\mathrm{m}, \mathrm{av}, e, z}^{(k)}
\end{array}\right]
\end{aligned}
$$

where

$$
\begin{aligned}
\overline{\bar{\nu}}_{\mathrm{d}, \mathrm{av}, e}^{(k)} & =\frac{1}{\ell_{r}} \int_{r_{1}}^{r_{2}}\left[\begin{array}{cc}
\nu_{\mathrm{d}, \theta \theta}^{(k)} \frac{r_{\mathrm{eq}}}{r} & \nu_{\mathrm{d}, z \theta}^{(k)} \\
\nu_{\mathrm{d}, \theta z}^{(k)} & \nu_{\mathrm{d}, z z}^{(k)} \frac{r}{r_{\mathrm{eq}}}
\end{array}\right] \\
{\left[\begin{array}{c}
H_{\mathrm{m}, \mathrm{av}, e, \theta}^{(k)} \\
H_{\mathrm{m}, \mathrm{av}, e, z}^{(k)}
\end{array}\right] } & =\frac{1}{\ell_{r}} \int_{r_{1}}^{r_{2}}\left[\begin{array}{c}
H_{\mathrm{m}, \theta}^{(k)} \\
H_{\mathrm{m}, z}^{(k)} \frac{r}{r_{\mathrm{eq}}}
\end{array}\right] \mathrm{d} r .
\end{aligned}
$$

The integrals in (15) and (16) are carried out by a onedimensional Gauss quadrature rule using the material points as quadrature points. The elementary 3 -by-3 matrices $\mathbf{K}_{\nu_{\mathrm{d}}, e, i j}$ and the elementary 3 -by-1 vectors $\widehat{\mathbf{j}}_{e, i}$ and $\widehat{\mathbf{j}}_{\mathrm{m}, e, i}$ are assembled into $\mathbf{K}_{\nu_{\mathrm{d}}}, \widehat{\mathbf{j}}$ and $\widehat{\mathbf{j}}_{\mathrm{m}}$.

In saturation, the originally assumed radial material symmetry is lost. Then, the magnetic flux migrates from one cylindrical shell to another, even if the geometry is fully radially symmetric [5]. Still, the choice of $\mathbf{w}_{e, j}$ imposes specific field symmetries for each intermediate solution. The magnetic flux density obeys

$$
\mathbf{B}(r, \theta, z)=\left(0, B_{\mathrm{eq}, \theta} \frac{r_{\mathrm{eq}}}{r}, B_{\mathrm{eq}, z}\right),
$$

when $\mathbf{B}\left(r_{\mathrm{eq}}, \theta, z\right)=\left(0, B_{\mathrm{eq}, \theta}, B_{\mathrm{eq}, z}\right)$. Equations (15) and (16) represent an averaging of $\overline{\bar{\nu}}_{\mathrm{d}}^{(k)}$ and $\mathbf{H}_{\mathrm{m}}^{(k)}$ in the radial direction. Notice that the individual components are averaged in a different way. The weighted residual approach averages
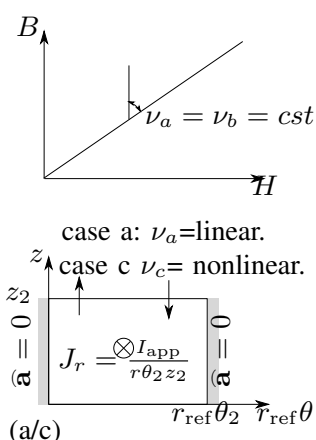

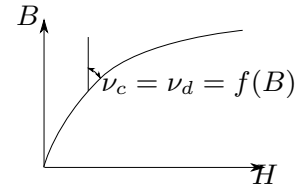

case b: $\nu_{b}=$ linear

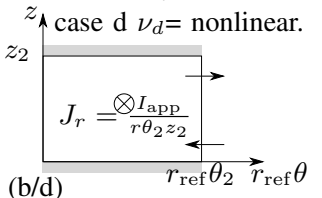

Fig. 4: Semi-analytical test cases.

the $\theta$ - and $z$-components of the magnetic field strength at the $(r, z)$ - and $(r, \theta)$-planes respectively, i.e.,

$$
\begin{aligned}
& H_{\mathrm{av}, \theta}=\frac{1}{\ell_{r} \ell_{z}} \int_{r_{1}}^{r_{2}} \int_{z_{1}}^{z_{2}} H_{\theta} \mathrm{d} r \mathrm{~d} z ; \\
& H_{\mathrm{av}, z}=\frac{1}{\ell_{r} r_{\mathrm{eq}} \ell_{\theta}} \int_{r_{1}}^{r_{2}} \int_{\theta_{1}}^{\theta_{2}} H_{z} r \mathrm{~d} r \mathrm{~d} \theta,
\end{aligned}
$$

where $\ell_{z}$ and $\ell_{\theta}$ are the extents of the considered planes in the $z$ - and $\theta$-direction. Inserting (2) and (17) into (18) and (19) leads to (15). An intuitive explanation is as follows. $B_{\theta}$ depends on $\frac{1}{r}$, whereas $B_{z}$ does not depend on $r$. Moreover, $H_{\theta}$ is averaged at $r z$-planes, whereas $H_{z}$ is averaged at $r \theta$-planes of which the cross-section depends on $r$. As a consequence, $\overline{\bar{\nu}}_{\mathrm{d}, \mathrm{av}, \theta \theta}$ is found by averaging $\overline{\bar{\nu}}_{\mathrm{d}, \theta \theta}$ scaled by

$\frac{r_{\mathrm{eq}}}{r}$, whereas $\overline{\bar{\nu}}_{\mathrm{d}, \mathrm{av}, z z}$ is found by averaging $\overline{\bar{\nu}}_{\mathrm{d}, z z}$ scaled by $\frac{r}{r_{\mathrm{eq}}}$. For the $\theta z$ - and $z \theta$-components of $\overline{\bar{\nu}}_{\mathrm{d}}$, the scaling factors cancel each other. The averaging of $\mathbf{H}_{\mathrm{m}}$ only affects its $z$ component.

\section{VALIDATION}

The correctness of the above nonlinear FE solver with radial symmetry is verified for 4 test cases. Each model considers a ring segment with rectangular cross-section, i.e., $V=\left[r_{1}, r_{2}\right] \times\left[0, \theta_{2}\right] \times\left[0, z_{2}\right]$, and is excited by a radial current. Test cases $a$ and $b$ contain a linear material, whereas test cases $c$ and $d$ contain a nonlinear material. The boundary conditions of test cases $a$ and $c$ only allow an axial flux, whereas test cases $b$ and $d$ only allow a peripheral flux. The validation is done by comparing the calculated magnetic energies (Table I). The linear models allow a comparison to analytical results [6]. For the nonlinear cases, the magnetic flux density has a simple spatial distribution, which allows to compute the magnetic energy according to the $\mathrm{BH}$-characteristic in a semi-analytical way. In the linear cases, an exact agreement is achieved. For the nonlinear cases, a distinction is made between the case with axial flux and the one with peripheral flux. The spatial distribution of the axial flux, and hence the saturation of the material, does not depend on $r$. Then, the nonlinear model preserves the radial material symmetry and the numerical and semi-analytical results match very well. On the other hand, a peripheral magnetic flux density is inhomogeneous in the radial direction and causes different levels of saturation. Then, the radial material symmetry is lost and results obtained by the $2 \mathrm{D}$ solver do no longer match the semi-analytical ones. 
TABLE I: Magnetic energy calculated by the 2D FE solver for models with radial symmetry compared to analytical or semi-analytical results.

\begin{tabular}{|l|r|r|}
\hline & 2D FE rad.symm. solver & (semi-)analytical \\
\hline linear material, axial flux & $6.535 e-07 \mathrm{~J}$ & $6.540 e-07 \mathrm{~J}$ \\
\hline linear material, peripheral flux & $8.491 e-06 \mathrm{~J}$ & $8.492 e-06 \mathrm{~J}$ \\
\hline nonlinear material, axial flux & $4.819 e-06 \mathrm{~J}$ & $4.821 e-06 \mathrm{~J}$ \\
\hline nonlinear material, peripheral flux & $1.561 e-05 \mathrm{~J}$ & $1.713 e-05 \mathrm{~J}$ \\
\hline
\end{tabular}

3D model
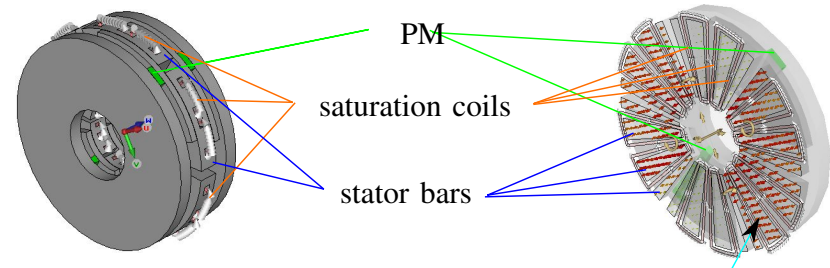

2D simulation (radial symmetry) saturated material

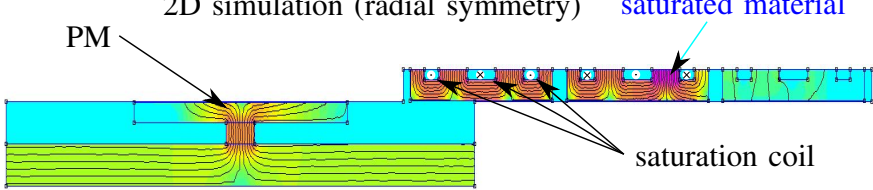

Fig. 5: Axial dual-rotor PM axial-flux machine with saturation coils.

\section{APPLICATION}

The nonlinear FE solver for 2D models with radial symmetry is now illustrated by calculating the performance of a dual-rotor permanent-magnet (PM) axial-flux machine with saturation coils (Fig. 5). The stator contains 6 coils that locally saturate the iron yoke at appropriate stator bars. The two rotors at the front and the back of the machine are shifted over 180 degrees, such that opposing magnetic poles are facing each other. The 2D model with radial symmetry is constructed in FEMM [16], triangulated by Triangle [17] and solved by a Matlab implementation of the new 2D nonlinear FE solver. Similarly as in the case of a 2D cartesian model, only the magnetically active part situated between $r=r_{1}$ and $r=r_{2}$ is considered (Fig. 2). Hence, end-winding effects are neglected. The 2D FE model serves for calculating electromotive forces and torques. End-winding effects can be modelled by additional resistances and inductances in an accompanying circuit model. The magnetic energy and torque of the motor are plotted as a function of the rotor position in Fig. 6.

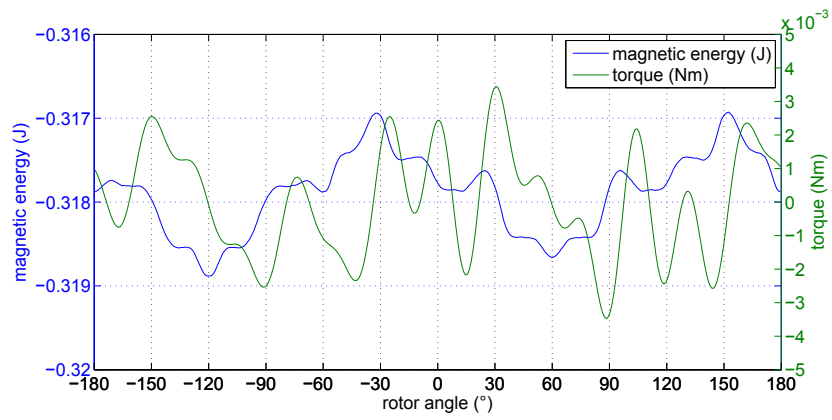

Fig. 6: Magnetic energy and torque as a function of the rotor position of the dual-rotor PM axial-flux machine.

\section{CONCLUSION}

A 2D magnetostatic FE solver for models with radial symmetry requires dedicated edge shape functions which also depend on the radial coordinate, necessitating a nonlinear implementation to consider several material points distributed along the direction of symmetry. Such situation is not encountered in the cartesian and axisymmetric 2D cases. The different saturation levels require material parameters to be averaged in the radial direction before being assembled into the system of equations in each Newton step. The solver has been validated for theoretical models and has been illustrated for a dual-rotor PM axial-flux machine.

\section{ACKNOWLEDGEMENTS}

This doctoral research is funded by the "Agency for Innovation by Science and Technology in Flanders (IWT)".

\section{REFERENCES}

[1] Y. H. Kim, S. Tao, S. O. Kwon, and J. P. Hong, "Influence of materials on design of interior PM synchronous motor," in ICEMS, Incheon, South Korea, Oct. 2010, pp. 1230-1234.

[2] F. I. Hantila, G. Preda, and M. Vasiliu, "Polarization method for static fields," IEEE Trans. Magn., vol. 36, no. 4, pp. 672-675, Jul. 2000

[3] E. Dlala and A. Arkkio, "Analysis of the convergence of the fixed-point method used for solving nonlinear rotational magnetic field problems," IEEE Trans. Magn., vol. 44, no. 4, pp. 473-478, Apr. 2008.

[4] P. Silvester and M. Chari, "Finite element solution of saturable magnetic field problems," IEEE Trans. Power Apparatus and Systems, vol. 89, no. 7, pp. 1642-1648, 1970.

[5] H. Vansompel, P. Sergeant, and L. Dupré, "A multilayer 2-D-2-D coupled model for eddy current calculation in the rotor of an axialflux PM machine," IEEE Trans. Energy Convers., vol. 27, no. 3, pp. 784-791, Sep. 2012.

[6] D. Vanoost, H. De Gersem, J. Peuteman, G. Gielen, and D. Pissoort, "2D magnetostatic finite element simulation for devices with a radial symmetry," submitted to IEEE Trans. Magn.

[7] — "Finite-element discretisation of the eddy-current term in a 2D solver for radially symmetric models," accepted for publication in Int. J. Numer. Model.

[8] H. De Gersem, I. Munteanu, and T. Weiland, "Construction of differential material matrices for the orthogonal finite-integration technique with nonlinear materials," IEEE Trans. Magn., vol. 44, no. 6, pp. 710-713, Jun. 2008.

[9] J. Azzouzi, G. Barakat, and B. Dakyo, "Quasi-3-D analytical modelling of the magnetic field of an axial flux permanent-magnet synchronous machine," IEEE Trans. Energy Convers., vol. 20, no. 4, p. 746-752, 2005.

[10] O. Niemimäki, "Improved quasi 3D modelling and simulation of axial flux machines," MSc Thesis, Tampere University of Technology, 2012. [Online]. Available: http://dspace.cc.tut.fi/dpub/bitstream/handle/ 123456789/21168/niemimaki.pdf

[11] P. Raumonen, S. Suuriniemi, T. Tarhasaari, and L. Kettunen, "Dimensional reduction in electromagnetic boundary value problems," IEEE Trans. Magn., vol. 44, no. 6, pp. 1146-1149, Jun. 2008.

[12] F. Henrotte, B. Meys, H. Hedia, P. Dular, and W. Legros, "Finite element modelling with transformation techniques," IEEE Trans. Magn., vol. 35 , no. 3, pp. 1434-1437, May 1999.

[13] J. C. Nédélec, "Mixed finite elements in $R^{3}$," Numer. Math., vol. 35, pp. 315-341, 1980.

[14] T. Weiland, "Time domain electromagnetic field computation with finite difference methods," Int. J. Numer. Model., vol. 9, no. 4, pp. 295-319, July-August 1996.

[15] E. Kubatko, B. Yeager, and A. Maggi, "New computationally efficient quadrature formulas for triangular prism elements," Computers \& Fluids, vol. 73, p. 187-201, 2013

[16] D. Meeker, "Finite element method magnetics (FEMM)," www.femm. info/wiki/HomePage.

[17] J. Shewchuk, "Delaunay refinement algorithms for triangular mesh generation," Computational Geometry, vol. 22, no. 1-3, pp. 21-74, May 2002. 